\title{
Assistência de enfermagem no pré-natal de baixo risco na atenção primária
}

\author{
Low-risk prenatal nursing care in primary care
}

Los cuidados de enfermería no prenatal de bajo riesgo en atención primaria

\author{
Aline Sharlon Maciel Batista RAMOS ${ }^{(1)}$ \\ Francisca das Chagas Gaspar ROCHA ${ }^{(2)}$ \\ Fernanda de Fátima Santos MUNIZ ${ }^{(1)}$ \\ Simony Fabíola Lopes NUNES ${ }^{(3)}$
}

Recebido: 1 dez 2016

Revisado: 11 maio 2017

Aceito: 28 jul 2017

Autor de

correspondência:

Aline Sharlon Maciel

Batista Ramos

alinesharlon@gmail.com

Conflito de interesses: Os autores declaram não haver nenhum interesse profissional ou pessoal que possa gerar conflito de interesses em relação a este manuscrito.
(1) Universidade CEUMA, São Luís, MA, Brasil.

(2) Centro Universitário UNINOFAFAPI, Teresina, PI, Brasil.

(3) Universidade Federal do Maranhão, UFMA, Imperatriz, MA, Brasil.

\section{Resumo}

O objetivo do estudo foi descrever as ações dos enfermeiros na atenção ao pré-natal de baixo risco em uma Unidade Básica de Saúde no interior do nordeste brasileiro. Trata-se de um estudo descritivo de natureza quantitativa, desenvolvido com 32 enfermeiros de uma Unidade Básica de Saúde no município de Santa Rita - MA, no período de setembro de 2014 a julho de 2015, sendo os dados coletados por meio de instrumento semiestruturado. Dos 32 enfermeiros, as principais ações realizadas eram: fornecer o cartão da gestante devidamente preenchido (84\%), identificar e classificar as gestantes de risco e/ou vulnerabilidade e encaminha gestantes de risco, para serviço especializado, respectivamente e $50 \%$ dos profissionais afirmaram que a dificuldade das gestantes é realizar os exames solicitados. Conclui-se que apesar do enfermeiro realizar as ações preconizadas no pré-natal de baixo risco, existem fatores que podem comprometer essas ações, como: a realização dos exames laboratoriais, a inexistência de capacitação e carência de protocolos na unidade.

Descritores: Cuidado Pré-Natal; Saúde da Mulher; Enfermagem Obstétrica.

\begin{abstract}
The objective of this study was to describe nursing actions in low risk prenatal care at a Basic Health Unit in the interior of the Brazilian Northeast. This is a descriptive study of a quantitative nature, developed with 32 nurses from a Basic Health Unit in the municipality of Santa Rita - MA, from September 2014 to July 2015, and the data were collected through a semi-structured instrument. Of the 32 nurses, the main actions carried out were: providing the pregnant woman's card filled out (84\%), identifying and classifying pregnant women at risk and / or vulnerability, and referring pregnant women to specialized services, respectively, and $50 \%$ That the difficulty for pregnant women is to perform the requested tests. It is concluded that although the nurse performs the actions recommended in low-risk prenatal care, there are factors that can compromise these actions, such as: laboratory tests, lack of training and lack of protocols in the unit.
\end{abstract}

Keywords: Prenatal Care; Women's Health; Obstetrics Nursing. 


\begin{abstract}
Resumen
El objetivo del estudio fue describir las acciones de enfermería en la atención a bajo riesgo prenatal en una Unidad Básica de Salud en el noreste de Brasil. Se trata de un estudio cuantitativo descriptivo, desarrollado con 32 enfermeros de una Unidad Básica de Salud en el municipio de Santa Rita - MA, desde septiembre 2014 hasta julio 2015, y los datos recogidos a través del instrumento semiestructurado. De las 32 enfermeras, las principales acciones llevadas a cabo fueron: para proporcionar la tarjeta embarazada completado (84\%), identificar y clasificar a las mujeres embarazadas corren el riesgo y / o vulnerabilidad y delanteros correr el riesgo de las mujeres embarazadas a servicios especializados, respectivamente, y el $50 \%$ de los profesionales de dicho la dificultad de las mujeres embarazadas es llevar a cabo las pruebas requeridas. Llegamos a la conclusión de que a pesar de la enfermera de realizar las acciones recomendadas en el bajo riesgo prenatal, hay factores que pueden comprometer estas acciones, como la realización de pruebas de laboratorio, la falta de formación y la falta de protocolos en la unidad.
\end{abstract}

Palabras-claves: Atención Prenatal; Salud de la Mujer; Enfermería Obstétrica.

\title{
Introdução
}

A assistência à saúde da mulher é destaque crescente no percurso das políticas de saúde no Brasil e foram implantadas em resposta à persistência de elevados coeficientes de mortalidade materna e perinatal. A atenção pré-natal permite o monitoramento da saúde da gestante, identifica fatores de risco e realiza a detecção e o tratamento oportuno de afecções, o que contribui para melhores desfechos maternos e perinatais. ${ }^{1}$

A rede básica compõe o primeiro nível de atendimento e desenvolve ações centradas na promoção à saúde e prevenção de doenças. ${ }^{2}$ Por isso, foi criado na década de 1990 o Programa de Saúde da Família - PSF, que passou em 1994 do status de programa para Estratégia Saúde da Família - ESF, precedido pelo Programa de Agentes Comunitários da Saúde - PACS, como forma de contemplar os princípios do Sistema Único de Saúde - SUS por meio do fortalecimento e consolidação da Atenção Básica. ${ }^{1,3}$

A implantação do PSF em todo o território nacional vem contribuindo positivamente para a melhoria de todos os indicadores de saúde, especialmente nas regiões onde as condições socioeconômicas e ambientais são mais precárias, como as regiões norte e nordeste nos quais se registram os maiores índices de mortalidade materna e infantil no Brasil. $^{1}$ 
A mortalidade materna no Brasil é 53 vezes maior que a observada em países desenvolvidos, sendo a relevância e o impacto desse problema agravado pelo alto índice de gravidez na adolescência, participação reduzida ou falta de adesão das gestantes ao programa de pré-natal, baixa escolaridade e contaminação pelo vírus da Síndrome da Imunodeficiência Adquirida - AIDS., ${ }^{4}$

O pré-natal na rede básica de saúde é realizado pelo enfermeiro e pelo médico e objetiva monitorar, prevenir e identificar intercorrências maternas e fetais e, ainda, realizar atividades educativas acerca da gravidez, parto e puerpério. No entanto, compete ao enfermeiro o acompanhamento das mulheres com ausência de complicações, cadastradas no pré-natal de baixo risco. ${ }^{6}$

O pré-natal é o acompanhamento da mulher grávida, desde o início da gestação que em $90 \%$ das mulheres é diagnosticado pela ausência de menstruação, e sintomas clássicos como enjoos e vômitos matinais, visando manter a integridade das condições de saúde da mãe e do bebê. Assim, durante toda a gravidez serão realizados exames e avaliações complementares com vistas a identificar e tratar precocemente as situações de risco que podem trazer prejuízos à saúde da mãe ou da criança. O que podem interferir no acompanhamento é a não adesão ao uso da medicação prescrita para o período gestacional de acordo com a necessidade, e as dificuldades em agendar/realizar os exames solicitados. ${ }^{7,8}$

As consultas do pré-natal de baixo risco devem ser realizadas mensalmente e apesar da gestação ser entendida como um processo fisiológico e que na grande maioria das vezes transcorre sem complicações, são preconizadas pelo Ministério da Saúde - MS, no mínimo seis consultas. ${ }^{9}$

Assim, as mulheres que não apresentam complicações no decorrer da gravidez são classificadas como grupo de gestantes de baixo risco e as que desenvolvem problemas durante o período gestacional ou evoluem com potenciais complicações para a mãe e feto compõe o grupo de gestantes de alto risco. ${ }^{10}$ 
O pré-natal de baixo risco realizado pelo enfermeiro objetiva monitorar e dar seguimento as gestantes de baixo risco, bem como, identificar adequada e precocemente as pacientes com potencial para evolução desfavorável, devendo ser encaminhadas para o acompanhamento de alto risco que é realizado pelo médico ginecologista. ${ }^{7}$

Frente ao exposto e a necessidade de aprimorar o conhecimento acerca da atuação do enfermeiro no pré-natal, justifica-se a relevância do presente estudo, por saber que o enfermeiro é peça importante na assistência, pois o mesmo assiste as gestantes durante a realização das consultas de pré-natal de baixo risco na rede básica de saúde.

Assim, constitui-se objetivo do presente estudo descrever as ações dos enfermeiros na atenção ao pré-natal de baixo risco em uma Unidade Básica de Saúde no interior do nordeste brasileiro.

\section{Metodologia}

Estudo descritivo, de natureza quantitativa no município de Santa Rita - MA. Santa Rita é um município do interior do Maranhão que margeia a BR-135, sendo esta o grande impulso econômico da região. É uma importante zona de produção agrícola, ativo centro de comércio e prestação de serviços, com população estimada de 36.500 pessoas, sendo que destes $61 \%$ reside na zona rural. O município é caracterizado por possuir 20 estabelecimentos de saúde cadastrados no SUS com atendimento ambulatorial e dois com atendimento obstétrico, e, possuir 14 equipes de estratégia de saúde da família.

Por envolver seres humanos, este estudo seguiu as normas nacionais e internacionais de ética em pesquisa com seres humanos e a coleta de dados iniciou-se após a aprovação pelo Comitê de Ética em Pesquisa da Universidade Ceuma (CAE n 43169015.5.0000.5084).

A coleta de dados ocorreu em ambiente privativo, nas dependências das unidades de saúde, individualmente com cada enfermeiro, conforme disponibilidade de horário dos profissionais, no período de setembro de 2014 a abril de 2015. O tipo de amostra foi de 
conveniência com a participação de 32 enfermeiros das Unidades Básicas de Saúde - UBS do munícipio.

Foram incluídos no estudo 32 enfermeiros que atendem diretamente no pré-natal.

Os dados foram obtidos por meio do uso de instrumento estruturado elaborado pelos autores com base no objetivo do estudo e em literatura específica, constituído de dados relacionados ao perfil sociodemográfico e ações de enfermagem realizadas pelos informantes. As variáveis coletadas contemplaram dados como: sexo, idade, escolaridade, estado civil, ocupação, renda e religião.

Os dados coletados foram inseridos no programa Microsoft Exce/®. A análise dos dados foi na forma descritiva. Para as variáveis qualitativas foi adotada frequência absoluta (n) e relativa (\%). Para as variáveis quantitativas foram empregadas como medidas-resumo: a média, a mediana e o desvio padrão (mínimo e máximo) para apontar a variabilidade dos dados.

\section{Resultados}

Dos 32 enfermeiros, $84 \%$ eram do sexo feminino e a média de idade da amostra investigada foi de 49,3 anos, sendo que $44 \%$ deles variavam dos 26 aos 30 anos. $\mathrm{Na}$ formação profissional, quanto ao tempo de formação, 46\% eram graduados em Enfermagem entre 4 a 5 anos; 54\% relataram ter realizado pelo menos um curso de especialização, sendo mais frequente o de Saúde da Família, conforme apresentado na Tabela 1. 
Tabela 1. Distribuição dos profissionais de enfermagem segundo dados sociodemográficos e de formação. Santa Rita - MA, Brasil. 2015.

\begin{tabular}{lcc}
\multicolumn{1}{c}{ Variáveis } & n & $\%$ \\
Idade & 4 & 13 \\
21 a 25 anos & 14 & 44 \\
26 a 30 anos & 3 & 9 \\
31 a 35 anos & 9 & 28 \\
36 anos a 40 anos & 2 & 6 \\
Mais de 40 anos & & \\
\hline Sexo & 27 & 84 \\
Feminino & 5 & 16 \\
Masculino & & \\
\hline Tempo de formação & 11 & 28 \\
1 a 3 anos & 18 & 46 \\
4 a 5 anos & 7 & 18 \\
6 a 8 anos & 3 & 8 \\
\hline 9 anos & & \\
\hline Especialização & $39^{*}$ & 100 \\
\hline Estratégia Saúde da Família & 21 & 54 \\
Saúde Pública/ Saúde Coletiva & 10 & 26 \\
Ginecologia e Obstetrícia & 4 & 10 \\
\hline Outros & 4 & 10 \\
\hline
\end{tabular}

${ }^{*}$ Total maior de 32, aceite de mais de uma alternativa.

Fonte: Elaboração própria a partir de dados coletados em campo (2015).

Em relação às ações realizadas pelos enfermeiros, destacam-se: fornecer o cartão da gestante devidamente preenchido (84\%), identificar e classificar gestantes de risco e/ou vulnerabilidade (84\%) e encaminhar gestantes de risco para serviço especializado (84\%) (Tabela 2). 
Tabela 2. Distribuição dos profissionais de enfermagem de acordo com os dados das ações realizadas pelos Enfermeiros no acompanhamento pré-natal. Santa Rita - MA, Brasil, 2015.

\begin{tabular}{|c|c|c|}
\hline Variáveis & $\mathbf{n}$ & $\%$ \\
\hline Realiza acolhimento & 24 & 75 \\
\hline Cadastramento da gestante no SISPRE NATAL WEB & 25 & 78 \\
\hline $\begin{array}{l}\text { Fornece o cartão da gestante devidamente } \\
\text { preenchido }\end{array}$ & 27 & 84 \\
\hline $\begin{array}{l}\text { Identifica e classifica gestantes de risco e/ou } \\
\text { vulnerabilidade }\end{array}$ & 27 & 84 \\
\hline $\begin{array}{l}\text { Encaminha gestantes de risco, para serviço } \\
\text { especializado }\end{array}$ & 27 & 84 \\
\hline Palestras e atividades educativas com a gestante & 25 & 78 \\
\hline $\begin{array}{l}\text { Palestras e atividades educativas com a gestante e } \\
\text { família }\end{array}$ & 21 & 66 \\
\hline $\begin{array}{l}\text { Consultas de Enfermagem intercalada com a } \\
\text { consulta médica }\end{array}$ & 25 & 78 \\
\hline $\begin{array}{l}\text { Solicitação de exames complementares de acordo } \\
\text { com o protocolo local e/ou do Ministério da Saúde }\end{array}$ & 26 & 81 \\
\hline $\begin{array}{l}\text { Realiza de teste rápido para HIV e triagem } \\
\text { sorológica para sífilis, Hepatite } B \text { e } C\end{array}$ & 15 & 47 \\
\hline $\begin{array}{l}\text { Prescreve medicamentos afixados nos protocolos de } \\
\text { pré-natal e abordagem sindrômica das ISTs }\end{array}$ & 22 & 54 \\
\hline Orienta e encaminha para imunização & 24 & 75 \\
\hline $\begin{array}{l}\text { Realiza exame clínico das mamas e colpocitologia } \\
\text { oncótica }\end{array}$ & 21 & 66 \\
\hline Realiza o pré-natal do homem & 9 & 28 \\
\hline
\end{tabular}

* ISTs: Infecção sexualmente transmissível

Fonte: elaboração própria a partir de dados coletados em campo (2015).

Dentre as principais dificuldades encontradas pelos enfermeiros no acompanhamento do pré-natal de baixo risco destacam-se dificuldade das gestantes em realizar os exames solicitados (50\%), a inexistência de cursos de capacitação (25\%), falta de protocolo na UBS, para o acompanhamento do pré-natal (22\%) e recursos materiais e insumos insuficientes (19\%), como representado na Tabela 3. 
Tabela 3 - Distribuição das dificuldades encontradas pelos enfermeiros no acompanhamento do pré-natal. Santa Rita - MA, Brasil, 2015.

\begin{tabular}{l|c|c}
\multicolumn{1}{c|}{ Variáveis } & $\mathbf{n}$ & $\%$ \\
$\begin{array}{l}\text { Não adesão das gestantes ao uso de } \\
\text { sulfato ferroso e ácido fólico }\end{array}$ & 6 & 19 \\
\hline $\begin{array}{l}\text { Dificuldade das gestantes em realizar os } \\
\text { exames solicitados }\end{array}$ & 16 & 50 \\
\hline $\begin{array}{l}\text { Não adesão das gestantes ao } \\
\text { acompanhamento pré-natal }\end{array}$ & 3 & 9 \\
\hline Recursos materiais e insumos insuficientes & 6 & 19 \\
\hline $\begin{array}{l}\text { Falta de protocolo na UBS, para o } \\
\text { acompanhamento pré-natal }\end{array}$ & 7 & 22 \\
\hline Recursos humanos insuficientes & 4 & 12 \\
\hline $\begin{array}{l}\text { Insegurança durante as consultas do pré- } \\
\text { natal }\end{array}$ & 3 & 9 \\
\hline \begin{tabular}{l} 
Inexistência de cursos de capacitação \\
\hline
\end{tabular}
\end{tabular}

Fonte: Elaboração própria a partir de dados coletados em campo (2015).

\section{Discussão}

O empreendimento de aferir o perfil e os aspectos dos enfermeiros no processo assistencial da atenção pré-natal transita pela precisão em ponderar e avaliar o panorama e as qualidades das atividades executadas, mediante a tomada de novas decisões. ${ }^{7,8}$

Os dados de caracterização dos enfermeiros que participaram deste estudo confirmaram o perfil esperado desta população. Prevaleceram enfermeiros do sexo feminino, seguindo as características históricas da profissão. Esse resultado ratifica o contexto histórico da Enfermagem, marcado pelo predomínio da força de trabalho feminina em atividades que envolvem o cuidado, explicitando a relação existente entre o fato de ser mulher e a opção pelos cursos de Enfermagem. ${ }^{11}$ 
O tempo de serviço é proporcional à exposição dos trabalhadores aos fatores de risco físico, biológico, ergonômico, psicossocial e químico, aspectos essenciais na saúde do trabalhador e na sua satisfação com o trabalho. ${ }^{5}$

No que diz respeito à formação dos enfermeiros que trabalham na atenção primária no atendimento ao pré-natal, resultados do estudo realizado no município de Montes Claros - MG, encontrou que $66,2 \%$ dos profissionais atuantes eram, somente graduados, comprovando ainda que os serviços de atenção primária a saúde (APS) que possuem profissionais com residência multiprofissional em saúde da família oferecem melhor desempenho na avaliação dos atributos da APS. No presente estudo, o tempo de serviço dos participantes foi em média de 1 a 3 anos, demonstrando que pode ter ocorrido uma mudança recente no perfil de trabalhadores destas UBS.

Neste estudo, os resultados evidenciaram que no cenário estudado, o cuidado prénatal é realizado por profissionais que possuem especialização em ESF, o que pode resultar em atendimento de alta qualidade em relação ao conteúdo das consultas, sendo esses profissionais, extremamente capacitados para a atenção ao pré-natal de baixo risco. Outros estudos sobre a qualidade do pré-natal corroboram esses resultados, apesar de os parâmetros avaliativos e metodológicos adotados não terem sido os mesmos. ${ }^{13}$

Isto se deve ao fato de que os profissionais da saúde e de outros setores têm buscado uma superespecialização, talvez influenciado pelo capitalismo, que exige trabalhadores com amplo conhecimento e polivalentes, para manter-se no mercado, levando-os a uma alienação trabalhista. $^{14}$

A estratégia governamental intitulada rede cegonha, implantada pela portaria $n^{\circ}$ 1.459 de 24/6/2011, 1,3 traz um modelo de atenção à Saúde Materno-infantil com assistência focada desde o planejamento reprodutivo até os dois anos de vida da criança. Exibindo padrão que oferece características de acolhimento e resolutividade, o plano objetiva o declínio da mortalidade materno-infantil de maneira que a efetividade dessa estratégia 
obtenha o principal objetivo proposto, estando os enfermeiros influentes como personalidades imperativas nesse processo. $^{3}$

O cuidado integral e humanizado, oferta de insumos necessários e a intervenção precoce diante certos sinais e sintomas realizados pela enfermagem devem contribuir de maneira significativa para que haja efetividade nos modelos preconizados pelo MS, propendendo à melhoria nos indicadores de morbi-mortalidade. ${ }^{15}$

Por sua vez, auxiliando tais indicadores, as ações educativas no decorrer do ciclo gravídico-puerperal, torna-se ferramenta importante, particularmente, no pré-natal, por orientar a mulher na futura vivência do parto de forma positiva, com menor risco de complicações no puerpério, desencadeando maior sucesso no cuidado com o bebê. ${ }^{16,17}$

Assim como existem políticas públicas que apontam a propriedade da assistência prénatal, existem determinados fatores que são imperativos para que isso ocorra, como: atendimento integral; a organização do serviço; a capacitação dos profissionais e a utilização de recursos adequados; e os requisitos básicos para promoção da saúde e prevenção das principais afecções. $^{16,17}$

Ressalta-se que a consulta pré-natal é um momento especial para o enfermeiro, onde o profissional pode desenvolver diversas ações inerentes a essa atividade com autonomia. Dentre as inúmeras atribuições do enfermeiro destaca-se a documentação em prontuário da história clínica e obstétrica, avaliação de risco gestacional, exame físico e obstétrico, orientação ao preparo para amamentação, orientação aos cuidados com a pele, ausculta dos batimentos cardiofetais, e ainda identificar e guiar sobre as queixas mais frequentes. ${ }^{18}$

Em estudo sobre dificuldades encontradas no pré-natal de baixo risco ${ }^{19,20}$ os enfermeiros apontaram o agendamento de exames o principal condicionante inibitório para um pré-natal de qualidade, sendo que em algumas unidades os exames de rotina das gestantes não eram realizados, apenas marcados para as mulheres realizarem na unidade sede de saúde do município, comprometendo a ida das gestantes. Já estudo realizado na Região da Zona Sul do Município de São Paulo ${ }^{8,10,21}$ evidenciou que falhas no registro de 
procedimentos, exames e imunização antitetânica comprometem a qualidade do pré-natal oferecido.

Algumas limitações podem ser reconhecidas no presente estudo, a exemplo, o estudo ser conduzido em um único município, não podendo realizar a generalização dos dados especialmente pela dimensão do país que leva a diferenças entre as regiões. Recomenda-se estudos sobre a temática com a associação entre a percepção dos profissionais, das gestantes e dados dos prontuários, a fim de promover maior avaliação dos serviços de saúde relacionados à assistência pré-natal.

\section{Conclusão}

A assistência pré-natal é fundamental para o preparo da maternidade e não deve ser encarada como simples assistência médica e sim, como trabalho de prevenção de intercorrências clínico-obstétricas e assistência emocional. A saúde da mulher deve ser considerada em sua totalidade, ultrapassando a condição biológica de reprodutora e conferindo-se o direito de participar globalmente das decisões que envolvem sua saúde.

A partir dos resultados encontrados, observou-se que alguns pontos impediam os enfermeiros de realizar as ações no pré-natal, os quais precisam de recursos para deliberação. Dentre esses recursos enquadram-se: o desenvolvimento de capacitações teóricas e práticas específicas, o provimento de informações quanto a importância da inclusão e uso de protocolos assistenciais e a criação de protocolos que promovam a interação do trabalho médico e de enfermagem com vistas à melhoria da qualidade dos serviços.

Portanto, deve-se ressaltar que os profissionais da área da saúde são responsáveis por uma grande parcela dos aspectos necessários para garantir a qualidade da atenção ao pré-natal. Para reordenar a estratégia de assistência de enfermagem, elaboraram-se protocolos de saúde. Estes são instrumentos criados para que os profissionais de saúde 
exerçam sua profissão de acordo com a regulamentação do exercício profissional. Através deles, os profissionais estarão normatizados e respaldados ao exercerem suas funções, zelando pela qualidade dos serviços prestados.

\section{Referências}

1. Brasil. Ministério da Saúde (BR). Atenção ao pré-natal de baixo risco. Brasília (DF): Ministério da Saúde; 2012.

2. Medeiros VC, Peres AM. Atividades de formação do enfermeiro no âmbito da atenção básica à saúde. Texto Context Enferm. 2011;20(no. spe):27-35. http://dx.doi.org/10.1590/S0104-07072011000500003.

3. Brasil. Ministério da Saúde (BR). Portaria no 1.459, de 24 de junho de 2011. Institui, no âmbito do Sistema Único de Saúde - SUS: a Rede Cegonha. Brasília, DF: Diário Oficial da União; 2011 [citado em 2 jan 2015]. Disponível em: http://bvsms.saude.gov.br/bvs/saudelegis/gm/2011/prt1459_24_06_2011.html.

4. Morse ML, Fonseca SC, Barbosa MD, Calil MB, Eyer FPC. Mortalidade materna no Brasil: o que mostra a produção científica nos últimos 30 anos? Cad Saude Publica. 2011;27(4):623-38. http://dx.doi.org/10.1590/S0102-311X2011000400002.

5. Souza SBCD, Tavares JP, Macedo ABT, Moreira PW, Lautert L. Influência do turno de trabalho e cronotipo na qualidade de vida dos trabalhadores de enfermagem. Rev Gaucha Enferm. 2012;33(4):79-85. http://dx.doi.org/10.1590/S1983-14472012000400010.

6. Costa CSC, Vila VC, Rodrigues FM, Martins CA, Pinho LMO. Características do atendimento pré-natal na Rede Básica de Saúde. Rev Eletron Enferm. 2013;15(2):516-22. http://dx.doi.org/10.5216/ree.v15i2.15635.

7. Vieira SM, Bock LF, Zocche DA, Pessota CU. Percepção das puérperas sobre a assistência prestada pela equipe de saúde no pré-natal. Texto Context Enferm. 2011;20(no. spe):255-62. http://dx.doi.org/10.1590/S0104-07072011000500032.

8. Corrêa MD, Tsunechiro MA, Lima MOP, Bonadio IC. Avaliação da assistência pré-natal em unidade com estratégia saúde da família. Rev Esc Enferm USP. 2014;48(no. spe):2331. http://dx.doi.org/10.1590/S0080-623420140000600004. 
9. Pio DAM, Oliveira MM. Educação em saúde para atenção à gestante: paralelo de experiências entre Brasil e Portugal. Saude Soc. 2014;23(1):313-24. http://dx.doi.org/10.1590/S0104-12902014000100025.

10. Macedo CPC, Barca DAAV, Valcácio FC, Dantas JA, Freitas MDLF, Costa ICC. Análise de dados do Sistema de Informação de Mortalidade (sim) e Comitê De Mortalidade Materna (CMM) no Rio Grande do Norte. Rev Bras Promoc Saude. 2013;25(4): 413-419. http://dx.doi.org/ 10.5020/18061230.2012.p413.

11. Duarte SJH, Almeida EP. O papel do enfermeiro do programa saúde da família no atendimento pré-natal. Rev Enferm Cent Oeste Min. 2014;4(1):1029-35. http://dx.doi.org/10.19175/recom.v0i0.137.

12. Matos DS, Rodrigues MS, Rodrigues TS. Atuação do enfermeiro na assistência ao prénatal de baixo risco na estratégia saúde da família em um município de Minas Gerais. Enferm Rev. 2013 [citada em 3 out 2016];16(1):18-33. Disponível em: http://periodicos.pucminas.br/index.php/enfermagemrevista/article/view/12937/10176.

13. Polgliane RBS, Leal MC, Amorim MHC, Zandonade E, Santos Neto ET. Adequação do processo de assistência pré-natal segundo critérios do Programa de Humanização do Pré-natal e Nascimento e da Organização Mundial de Saúde. Cienc Saude Colet. 2014;19(7):1999-2010. http://dx.doi.org/10.1590/1413-81232014197.08622013.

14. Silva CSO, Fonseca ADG, Souza LPS, Siqueira LG, Barbosa DA. Qualificação de enfermeiros em Saúde da Família e qualidade da atenção na ótica de usuários. Cienc Cuidado Saude. 2015;14(3):1251-8. http://dx.doi.org/10.4025/cienccuidsaude.v14i3.22934

15. Silva ALS, Nascimento ER, Coelho EAC, Nunes IM. Atividades educativas no pré-natal sob o olhar de mulheres grávidas. Rev Cubana Enferm. 2014 [citado em 3 out. 2016];30(1). Disponível em: http://www.revenfermeria.sld.cu/index.php/enf/article/view/487. Acesso em: 03 Out. 2016.

16. Barbieri MC, Soares NT, Ferrari, RAP, Demitto MO, Tacla MTGM. Vivência da maternidade: percepção de mulheres participantes de grupos de pré-natal. Rev Enferm UFPE. 2013;7(9):5533-40. https://doi.org/10.5205/1981-8963-v7i9a13671 p5533-55402013. 
17. Rocha RS, Silva MGC. Assistência pré-natal na rede básica de Fortaleza-CE: uma avaliação da estrutura, do processo e do resultado. Rev Bras Promoc Saude. 2013 [citado 3 out. 2016];25(3):344-55. Disponível em: http://www.redalyc.org/articulo.oa?id=40823864013.

18. Souza VB, Roecker S, Marcon SS. Ações educativas durante a assistência pré-natal: percepção de gestantes atendidas na rede básica de Maringá-PR. Rev Eletron Enferm. 2011;13(2):199-210. http://dx.doi.org/10.5216/ree.v13i2.10162.

19. Fontanella APS, Wisniewski D. Pré-natal de baixo risco: dificuldades encontradas pelos profissionais enfermeiros. Braz J Surg Clin Res. 2014;7(3):11-6.

20. Silva EP, Lima RT, Maria Osório M. Impacto de estratégias educacionais no pré-natal de baixo risco: revisão sistemática de ensaios clínicos randomizados. Cienc Saude Colet. 2016;21(9):2935-48. http://dx.doi.org/10.1590/1413-81232015219.01602015.

21. Teixeira E, Vale EG, Fernandes JD, Sordi MRL. Enfermagem. In: Haddad AE, Pierantoni CR, Ristoff D, Xavier IM, Giolo J, Silva LB, orgs. A trajetória dos cursos de graduação na área da saúde: 1991-2004. Brasília, DF: Instituto Nacional de Estudos e Pesquisas Educacionais Anísio Teixeira; 2006.

\section{Minicurrículo}

Aline Sharlon Maciel Batista Ramos | ORCiD: 0000-0002-7648-2857

Doutoranda em Ciências Médicas na Universidade Estadual do Rio de Janeiro - UERJ. Professora Assistente da Universidade CEUMA.

Francisca das Chagas Gaspar Rocha | ORCiD: 0003-1193-248X

Doutoranda em Engenharia Biomédica na Universidade Brasil. Professora Assistente da Universidade CEUMA.

\section{Fernanda de Fátima Santos Muniz}

Enfermeira. Graduada pela Universidade CEUMA.

Simony Fabíola Lopes Nunes | ORCiD: 0000-0003-4613-8542

Doutoranda em Enfermagem na Universidade Federal de Santa Catarina - UFSC. Professora Assistente da Universidade Federal do Maranhão - UFMA. 